\title{
Review of: "Genetic loci of the R. anatipestifer serotype discovered by Pan-GWAS and its application for the development of a multiplex PCR serotyping method"
}

Xi Guo

Potential competing interests: The author(s) declared that no potential competing interests exist.

The authors characterized the $\mathrm{O}$ antigen gene cluster of $\mathrm{R}$. anatipestifer, a poultry pathogen worldwide. Based on this, a multiplex PCR assay was developed targeting the major serotypes, which exhibited good agreement to the result generated from the antigenic method. I think the work provide a molecular serotyping scheme for R. anatipestifer and could facilitate the work on epidemiological surveillance. In general, the MS is well organized and written, and the method is clear. Meanwhile, I still have some question to be addressed by the authors.

Major concern:

The authors described the genetic loci discovered by Pan-GWAS as O-antigen clusters. However, it is always very similar between the O-AGC and the capsule gene cluster. And within the gene cluster, wza and wzc exist, which are always responsible for the transport of capsule along with wzb. Then, how to define them as the O-AGCs? On the other hand, are previous studies of the defined serotypes associated with Oantigen? The authors constructed a wzy mutant strain and tested it by using agglutination test, which partially identify the O-AGC. However, I think, this is not the ample evidence. As the existence of O-antigen is normally visualized by the lipopolysaccharide (LPS) profile.

Minor concerns:

1, line 54 to 55, at least 21 serotypes have been reported.... Among them, are there some dominant ones? As the multiplex PCR was developed against three serotypes (1, 2 and 11$)$.

2, line 55 to 56 , "There is no effective cross-protection among different serotypes". It seems sudden.

3 , in addition to the serotyping method, are there any other typing assays? What are the others advantages and disadvantages?

3, the O-antigen structure and the O-antigen gene cluster in Gram-negative bacteria should be introduced to the readers. Any O-antigen structures have been elucidated? 
4, among the strains listed in supplementary table 1, did the authors perform genome sequencing for some of them?

5, line 241 to 248, Except the Wzx/Wzy dependent pathway, the ABC transporter pathway (Wzm/Wzt dependent) is another important system for the O-antigen synthesis. However, it is not mentioned in this MS.

6, line 276, "rhamnose", should be L-rhamnose.

7, line 351, may be Figure 11. And line356, may be Figure 12. Delete Figure 12 on line 191.

8, line 184, what's the purpose of E.coli X7232?

9, to perform the multiplex assay, how to obtain the template? Did the author evaluate the PCR assay by using pure cultures? 